\title{
Los procesos de evaluación del aprendizaje en tiempos de pandemia. La percepción del profesorado del Colegio Las Condes de Santiago de Chile
}

\section{Learning assessment processes in times of pandemic. The perception of teachers at Las Condes School in Santiago, Chile}

DOI: $10.46932 / \mathrm{sfjdv} 2 \mathrm{n} 5-120$

Received in: Oct 1st, 2021

Accepted in: Dec 30th, 2021

\author{
Tulio Barrios Bulling \\ Doctor of Education, Warnborough College Dublin \\ Universidad Nacional Andrés Bello Chile \\ E-mail: t.barriosbulling@uandresbello.edu \\ Laura Barrios Valenzuela \\ Máster en Psicopedagogía, Universidad Alcalá de Henares, España \\ The International School La Serena, Chile \\ E-mail: barrioslaura9@gmail.com
}

\section{RESEÑA}

La presente investigación se realiza en el Colegio Las Condes de Santiago de Chile, en un momento en que el país se encuentra bajo medidas de confinamiento por la pandemia. Los establecimientos educacionales han debido establecer sistemas de enseñanza y evaluación en línea para dar continuidad al proceso educativo. Esta investigación indaga acerca de los cambios realizados a los procesos evaluativos y acerca de aquellos instrumentos de evaluación más utilizados y eficientes en dicho contexto. Mediante una encuesta semi estructurada que arrojó datos tanto cuantitativos como cualitativos, se ha podido determinar que los profesores han experimentado serias dificultades al afrontar los procesos evaluativos en línea. El diseño de instrumentos se volvió más personal y menos colaborativo. Los alumnos participan escasamente en dichos procesos. Los encuestados privilegiaron instrumentos de fácil aplicación, sencillos de corregir y que impactaran en la motivación de sus estudiantes. La insuficiente preparación de los profesores para evaluar en entornos virtuales, crea la necesidad de implementar sistemas de capacitación para fortalecer su manejo tecnológico. Urge la búsqueda de nuevos espacios de reflexión y colaboración que permitan mejorar las prácticas pedagógicas y los procedimientos de retroalimentación.

Palabras clave: Evaluación aprendizaje, cambio, instrumentos eficientes, pandemia

\begin{abstract}
This research is conducted at Colegio Las Condes in Santiago de Chile, at a time when the country is under confinement measures due to the pandemic. Educational establishments have had to establish online teaching and evaluation systems to give continuity to the educational process. This research inquires about the changes made to the evaluation processes and about the most used and efficient evaluation instruments in this context. Through a semi-structured survey that yielded both quantitative and qualitative data, it has been determined that teachers have experienced serious difficulties when facing online evaluation processes. Instrument design became more personal and less collaborative. Students barely participate in these processes. Respondents favoured instruments that were easy to apply, simple to correct and that impacted on the motivation of their students. The insufficient preparation of teachers to evaluate in virtual environments creates the need to implement training systems to strengthen their technological
\end{abstract}


management. There is an urgent need to search for new spaces for reflection and collaboration that allow the improvement of pedagogical practices and feedback procedures.

Keywords: Learning assessment, change, efficient instruments, pandemic

\section{INTRODUCCIÓN}

El brote de la pandemia conocida como COVID-19 se ha extendido por todo el mundo, afectando todos los países, sus ámbitos de desarrollo y, entre ellos, a la educación también. Esto ha obligado a los gobiernos a implementar medidas de prevención como el lavado frecuente de manos, el uso de mascarillas faciales, el distanciamiento físico y las reuniones de carácter masivo. Adicionalmente, se han implementado estrategias de encierro y cuarentenas como acciones necesarias para tratar de controlar y disminuir la transmisión de la enfermedad.

En el medio de esta situación, cada país ha tratado de darle continuidad a sus sistemas educativos de distintas maneras, de acuerdo a su realidad y recursos disponibles. En Chile, la mayoría de las escuelas y las universidades han reconvertido su infraestructura para darle continuidad a los procesos de enseñanzaaprendizaje con clases en línea. En períodos de mejora de la cantidad de nuevos casos de contagiados por el referido virus, algunos colegios han reabiertos sus aulas, pero con aforos reducidos y con un sistema híbrido de enseñanza. Este proceso ha sido complejo por la necesidad de mayores recursos tecnológicos, pero más aún por la necesidad de capacitar al profesorado en el uso de nuevas plataformas de enseñanza

y de evaluación del aprendizaje quienes, hasta antes de la pandemia, habían tenido una exposición limitada a la enseñanza en línea.

Si bien estos cambios pueden constituir también una oportunidad, pareciera existir una opinión generalizada, sustentada por algunas investigaciones preliminares (Soland J., Kuhfeld, Tarasawa, Johnson, Ruzek, y Liu, 2020; Engzella, Freya. y Verhagena 2021; Gore, Fray, Miller, Harris y Taggart 2021). que la calidad de los aprendizajes se ha visto afectada por la enseñanza en línea y que se hace muy complejo validar la confiabilidad de los procesos evaluativos. En consecuencia, este artículo busca indagar en aquellos instrumentos o técnicas de evaluación que, a juicio de los profesores del Colegio Las Condes de Santiago de Chile, han sido más utilizados y más eficientes y las razones para aquello.

\section{CONTEXTO}

Nuestra investigación se realiza a partir de la información recopilada de los profesores del Colegio Las Condes de Santiago de Chile. Este establecimiento de dependencia municipal, abre sus puertas el 27 de febrero del año 2021. 
El Colegio Las Condes busca ofrecer una educación de calidad e inclusiva con sello en la enseñanza del idioma inglés desde los primeros años de escolaridad. Actualmente acoge a 1068 estudiantes divididos en tres cursos por nivel desde Pre kínder hasta Cuarto Medio (año 12 de escolaridad). Adicionalmente, los cursos están funcionado bajo un sistema híbrido de clases presenciales y en línea, a fin de dar cumplimiento a las medidas sanitarias y los aforos de las salas determinados por las autoridades sanitarias. Esto ha implicado que la mitad de un curso atienda clases de manera presencial, al tiempo que la otra mitad sigue las clases desde sus casas con transmisiones en directo, para luego rotar a la semana siguiente.

Respecto al personal del colegio, este está compuesto por un Director, ocho miembros del equipo de gestión, divididos en las áreas académicas, de convivencia y orientación, 81 profesores, seis asistentes de la educación, siete paradocentes de apoyo a la convivencia, cinco funcionarios administrativos y siete auxiliares de aseo y servicios.

Los profesores provienen de distintas realidades educacionales, 75,3\% de ellos de colegios particulares pagados, $12,3 \%$ de colegios particulares subvencionados, 9,9\% de colegios públicos municipales y un $2,5 \%$ proceden directamente de la universidad. Un $32 \%$ corresponde a hombres, mientras que el $68 \%$ restante a mujeres.

Respecto a los años de experiencia, un $56,8 \%$ se encuentran en el rango de 0 a 5 años, un $16 \%$ entre 6 y 10 años, un 9,9\% entre 11 y 15 años, un 7,4\% entre 16 y 20 años, un 4,9\% entre 21 y 25 años, un 2,5\% entre 26 y 30 años y finalmente un 2,5\% con más de 30 años de experiencia. En lo concerniente a los a los ciclos en que se desempeñan, un $21 \%$ pertenecen al ciclo Preescolar, un $28 \%$ al ciclo Básico y un $51 \%$ a Educación Media.

\section{REVISIÓN DE LA LITERATURA}

Con el propósito de darle a nuestra investigación el necesario sustento teórico, revisaremos algunos aportes a los temas en estudio desde la literatura especializada.

\subsection{LA EDUCACIÓN EN TIEMPOS DE PANDEMIA}

Al momento del presente estudio, cifras de la UNESCO (2020) reportan que 1600 millones de estudiantes de 190 países habían dejado de asistir a clases cuando la pandemia alcanzó el máximo informado de casos de contagio. A mayor abundamiento, más de 100 millones de docentes y de personal escolar se han visto afectados por el cierre de establecimientos educativos. Esto sigue impactando de forma parcial o total, aún al día de hoy, a dos tercios de la población estudiantil del mundo. 
Respecto a Latinoamérica, CEPAL (2020) estima que el impacto económico de la pandemia será más severo en esta región debido a la vulnerabilidad de las economías locales. Además, muchos países también enfrentan problemas de gobernanza.

En Chile, las clases presenciales se suspenden y, con el fin de dar continuidad al proceso de enseñanza aprendizaje, el Ministerio de Educación (MINEDUC) realiza una priorización curricular. Los establecimientos educacionales suben sus programas a plataformas, desde las cuales intentan, con clases sincrónicas y actividades asincrónicas, dan cumplimiento a los objetivos y contenidos prioritarios. Esta situación pone en evidencia, de acuerdo a Murillo y Duk (2020), la desigualdad en conectividad, la falta de preparación de los profesores para funcionar en entornos virtuales, la falta de autonomía de los estudiantes y la necesidad de contar con un apoyo más directo de padres y apoderados.

\subsection{APRENDIZAJE}

El aprendizaje ha sido históricamente uno de los conceptos más estudiados debido a la importancia que posee para los procesos tanto cognitivos como pedagógicos. Es así como permanentemente recibe aportes desde distintas áreas del saber.

Mayer (2003) considera el aprendizaje como "el cambio relativamente permanente en el conocimiento de una persona o comportamiento debido a la experiencia." Esta definición es muy cercana a los postulados de Gagné (1965), debido a que también considera que la duración del cambio debe ser a largo plazo y que este se produce como la resultante del intercambio de un individuo con su ambiente y no del mero desarrollo o crecimiento biológico. De igual forma, Driscoll (2005) asocia el crecimiento a un cambio perdurable en lo que él llama "el rendimiento humano", el cual también derivaría de la interacción de un sujeto con tu medio o entorno.

Pimienta (2008) le da a la educación un rol influyente en el aprendizaje, ya que lo considera como “el conjunto de productos obtenidos por los estudiantes como resultado de la incidencia de la educación." (p. 26) Vemos como este autor introduce la noción de productos a su definición, lo que es consistente con el enfoque o modelo de educación por competencias.

Una concepción más compleja de aprendizaje es la entregan Bingham y Conner (2010). Para estos autores, el aprendizaje es un proceso de transformación en la forma como absorbemos la información. Este proceso pasa por una etapa de interiorización que interactúa con nuestras experiencias, cambiando así nuestros conocimientos basados en lo que hacemos. Para Bingham y Conner el aprendizaje se basa en insumos, procesos y la reflexión. Esta visión procesual también es compartida por Ambrose, Bridges, Di Pietro, Lovett, y Norman (2010) quienes postulan que el aprendizaje es "un proceso que conduce a un cambio, que se produce como resultado de la experiencia y aumenta el potencial de mejorar el rendimiento y el aprendizaje futuro". (p.3) De esta definición se puede desprender que Ambrose et al. consideran el 
aprendizaje como un proceso, no como un producto, que el aprendizaje involucra cambios y que también depende de la experiencia de cada individuo.

Brown, Roedinger y McDaniel (2014) conciben derechamente el aprendizaje como la adquisición de conocimientos y habilidades. Además, para que este aprendizaje se produzca tanto los conocimientos como las habilidades deben estar disponibles en la memoria para poder resolver problemas y oportunidades futuras. Esta concepción de aprendizaje tiene implicaciones pedagógicas directas, es especial a lo que a estrategias de enseñanza y evaluación de los aprendizajes se refiere. Hernández, Pérez y Quiroz (2021) consideran que el "aprendizaje es un proceso corresponsable, participativo y permanente, donde intervienen: el aprendiz, docente, condiscípulos, institución y demás factores sociales donde se desenvuelve el estudiante". (p. 7086) De esta forma, el aprendizaje sería un proceso multifactorial donde intervienen distintos agentes y ambientes.

A modo de síntesis podemos señalar que el concepto de aprendizaje es complejo, por lo que no existe una definición ampliamente aceptada y validada. Cada autor aporta desde su especialidad, lo que da lugar a diversos enfoques y a la necesidad de integrar las distintas disciplinas que estudian el aprendizaje. Los elementos comunes que comparten las distintas visiones del aprendizaje parecieran ser el proceso, la necesidad de un sujeto que aprende, la experiencia que media el aprendizaje y el cambio de conocimiento que genera el aprendiz.

\subsection{EVALUACIÓN DEL APRENDIZAJE}

Tras el acercamiento al concepto de aprendizaje recientemente realizado, pasaremos a revisar algunos conceptos relacionados a su evaluación. Tradicionalmente, la evaluación la tenido como objetivo indagar e informar sobre lo aprendido. De ahí su relación con las estrategias de enseñanza-aprendizaje y su función mediadora en los profesores y sus estudiantes. Es así como Amua-Sekyi (2016) define la evaluación como "todas las actividades que los profesores y estudiantes realizan para obtener información que pueda utilizarse para alterar la enseñanza y el aprendizaje.” (p. 1) Esta definición incluye tareas de observación y análisis del trabajo de los alumnos con el propósito de obtener evidencia acerca de lo que ellos saben o pueden hacer.

Dependiendo de los usos que se les den a los procesos evaluativos, la evaluación se categoriza en formativa o sumativa (Dixson y Worrell, 2016). La evaluación formativa está integrada en el proceso de enseñanza y aprendizaje y proporciona retroalimentación al profesor en el curso de enseñanza para permitirle juzgar qué tan bien están aprendiendo los estudiantes. También proporciona información sobre la eficacia de la enseñanza que ayudará a determinar una acción correctiva apropiada cuando sea necesario. La evaluación sumativa, por su parte, se lleva a cabo al final de una unidad, curso o programa para determinar el nivel de logro de los estudiantes. En ocasiones, señalan Lecrecq y Cabrera (2014), 
puede darse el caso donde se empleen ambos tipos de evaluación, generando así una situación evaluativa mixta. En esta misma línea, Mateo y Martínez (2008) dividen los procedimientos evaluativos en tres tipos, tradicionales, mixtos y alternativos. En el primer tipo de procedimiento evaluativo, los autores identifican las pruebas objetivas, las pruebas escritas o de ensayo corresponderían a la tipología mixtas, mientras que el uso del portafolio, el desarrollo de proyectos y ejecuciones en contexto de simulación corresponderían a los sistemas alternativos.

Una visión de la evaluación de los aprendizajes que viene ganando el interés de autores e investigadores, es el concepto de evaluación para el aprendizaje. Esta concepción requiere que los profesores y los alumnos utilicen la evaluación para mejorar tanto la instrucción y como el aprendizaje. Se trata de evaluar el progreso de los alumnos para entregarles retroalimentación y tomar decisiones acerca de las siguientes etapas del proceso de enseñanza y aprendizaje. Ya no se trata de certificar el aprendizaje sino más bien en mejorarlo. El uso de la evaluación para el aprendizaje puede ayudar a los profesores a cumplir con otros componentes del aprendizaje como son las habilidades de pensamiento, el desarrollo personal, el aprendizaje permanente y la comprensión mutua.

Desde una perspectiva socio-cultural, Sardareh y Mohd Saad (2013) definen la evaluación para el aprendizaje como "todas las prácticas durante el proceso de enseñanza y aprendizaje que tienen como objetivo mejorar la enseñanza y el aprendizaje y ayudar a los estudiantes a convertirse en dueños de su propio aprendizaje.” (p. 2496) Esta definición pone de manifiesto la necesaria interacción profesoralumno durante el proceso evaluativo que debe llevar al estudiante a tomar control de su proceso de aprendizaje. Al participar de su evaluación, se crean las condiciones necesarias para que el alumno alcance la autonomía y la capacidad de gestionar su aprendizaje.

Una postura similar presenta el Western and Northern Canadian Protocol for Collaboration in Education (2006). En palabras de sus autores

\footnotetext{
Cuando el aprendizaje es el objetivo, los profesores y los estudiantes colaboran y utilizan la evaluación y retroalimentación pertinente para mover el aprendizaje hacia adelante. Cuando la evaluación en el aula es frecuente y variada, los profesores pueden aprender mucho sobre sus estudiantes. Pueden ganar en comprensión de las creencias existentes dentro de sus estudiantes y de su conocimiento y pueden identificar entendimientos incompletos, creencias falsas e interpretaciones ingenuas de conceptos que pueden influir o distorsionar el aprendizaje. Los profesores pueden observar y sondear el pensamiento de los estudiantes a lo largo del tiempo e identificar vínculos entre conocimientos previos y el nuevo aprendizaje. (p. 5)
}

La evaluación para el aprendizaje también invita a los estudiantes a pensar en sus propios aprendizajes. Para ellos, los alumnos deben revisar sus experiencias de aprendizaje y gestionar las estrategias para aplicar lo aprendido a situaciones de aprendizaje futuras. La evaluación proporciona la retroalimentación para este proceso. Cuando los estudiantes y sus profesores se involucran en un ciclo continuo de retroalimentación y ajustes, el aprendizaje se vuelve más eficiente y los estudiantes comienzan 
a internalizar el proceso de gestión. Cuando los estudiantes se hacen partícipes de esta experiencia evaluativa continua, logran monitorear su aprendizaje, hacer correcciones y desarrollar el hábito de revisar y desafiar continuamente lo aprendido.

\section{OBJETIVOS}

Los objetivos que guían y orientan la presente investigación son:

- Conocer los cambios producidos en la evaluación de los aprendizajes por la educación en línea

- Comprender sus usos y finalidades.

- Identificar los instrumentos de evaluación más utilizados y más efectivos en este contexto.

- Detectar las principales dificultades y desafíos.

\section{METODOLOGÍA}

Dado el contexto de pandemia, diseñamos una encuesta semi estructurada (preguntas de alternativas y otras abiertas) utilizando la plataforma Google Forms, la cual fue sometida previamente a una prueba piloto. Tras revisar el proceso de pilotaje, realizamos algunos ajustes al instrumento. Luego invitamos a ser parte del estudio a 57 profesores del área científico-humanista. La participación fue anónima y voluntaria. 43 profesionales contestaron la encuesta, lo que constituye el 75,4\% del total.

Los datos de carácter cuantitativo fueron procesados mediante una planilla de cálculo Excel y graficados para facilitar la visualización del análisis. Los datos cualitativos obtenidos a través de las preguntas abiertas, fueron estudiados por ejes temáticos.

\section{ANÁLISIS DE LOS DATOS}

A continuación, presentaremos el análisis de los datos cuantitativos recogidos a través de la encuesta aplicada.

\subsection{DISEÑO DE LOS INSTRUMENTOS DE EVALUACIÓN}

Consultados respecto a quién o quiénes diseñan los instrumentos de evaluación, los profesores informantes arrojaron los siguientes datos que se pueden visualizar el la Figura 1. 
Figura 1. Gráfico Diseño de los instrumentos de evaluación

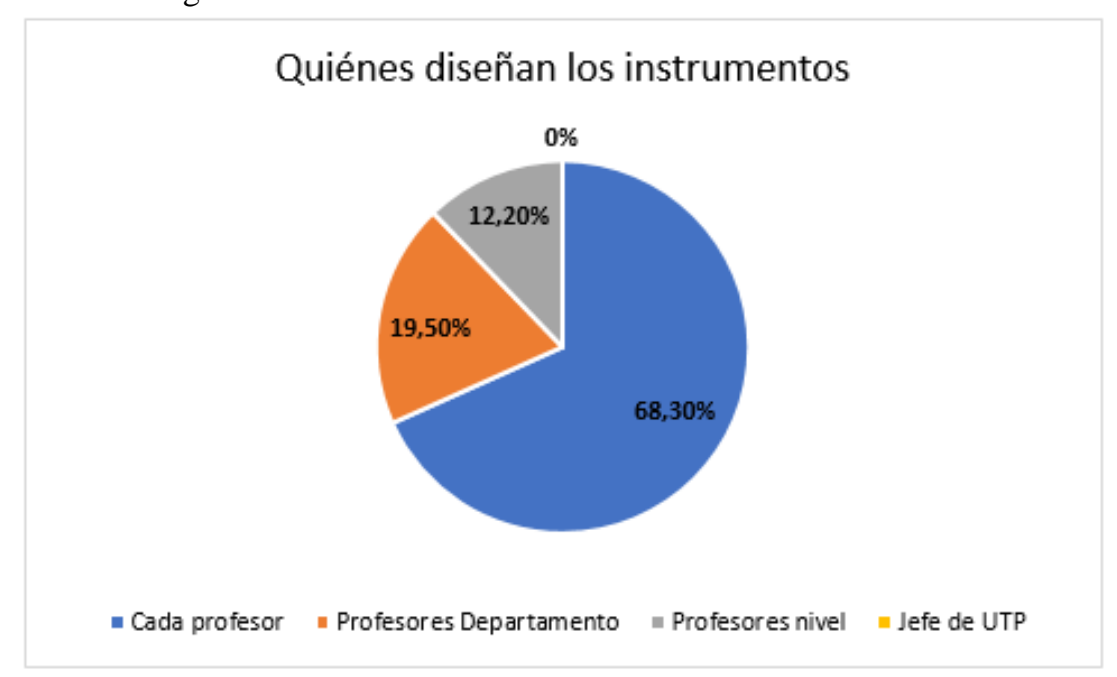

La gran mayoría de los encuestados $(68,3 \%)$ reporta que el proceso de diseño de los instrumentos de evaluación es personal. Un 19,5\% informa acerca de un proceso colaborativo entre profesores de un mismo departamento, mientras que un $12,2 \%$ señala que se trata de un proceso que llevan a cabo los docentes de un mismo nivel. La unidad técnico pedagógica (UTP) no tendría participación alguna en este proceso de acuerdo a los integrantes de la muestra.

\subsection{COMPARTICIÓN DE LAS PRÁCTICAS EVALUATIVAS}

Ante la pregunta si se compartían las prácticas evaluativas y con qué frecuencia, el 24, 4\% de los profesores reportaron que siempre se compartían, un 29,3\% de ellos que la compartición sucedía frecuentemente y para $31,7 \%$ de los informantes, esto se efectuaba solo de manera ocasional. El 14,6\% de la muestra reporta no haber compartido las prácticas evaluativas. Toda esta situación se puede ver en la Figura 2.

Figura 2. Gráfico Compartición de prácticas evaluativas

\section{Compartición de prácticas evaluativas}

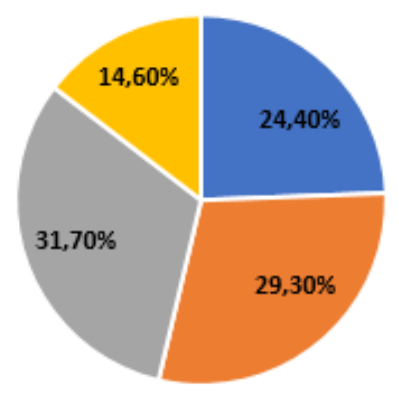

- Siempre | Frecuentemente | Ocasionalmente " Nunca 


\subsection{PROMOCIÓN DE UNA EVALUACIÓN DIVERSIFICADA}

Respondiendo a la pregunta si sus anteriores escuelas promovían una evaluación diversificada. El $31,7 \%$ de los profesores informantes señalan que, si lo hacían y de manera decidida, para el 48,8\% esto sucedía, pero de manera moderada; mientras que para el 19,5\% de los informantes, esto no sucedió en sus comunidades educativas. La Figura 3 nos permite apreciar esta situación.

Figura 3. Gráfico Promoción de una evaluación diversificada

Promoción evaluación diversificada

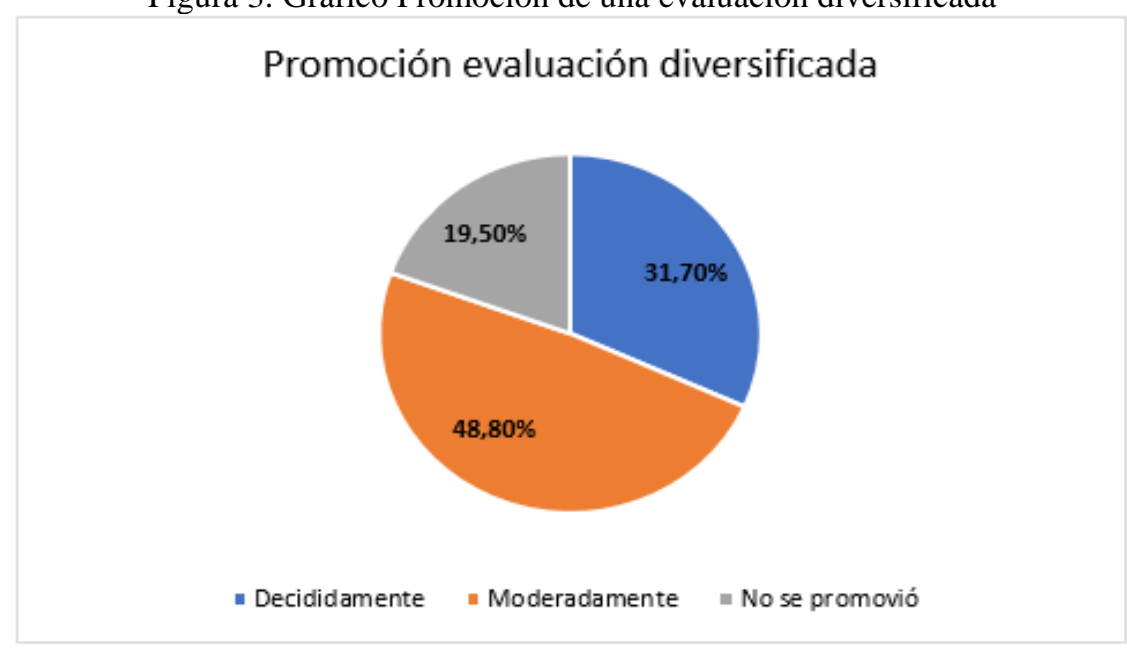

\subsection{NECESIDAD DE FORMACIÓN EN EVALUACIÓN DIVERSIFICADA}

Todos los profesores encuestados reconocen alguna necesidad de mayor formación en evaluación diversificada. El 51,2\% de ellos en evaluación para entornos en línea, mientras que el 48,8\% restante, para cualquier tipo de contexto.

\subsection{USO DE RESULTADOS DE LA EVALUACIÓN PARA MODIFICAR LA PRÁCTICA DOCENTE}

La Figura 4 presenta las respuestas obtenidas a la pregunta si se utilizan los resultados de aprendizaje de los estudiantes para modificar la práctica docente.

Figura 4. Gráfico Modificación de práctica docente a partir de los resultados

Uso de resultados evaluación para modificar práctica
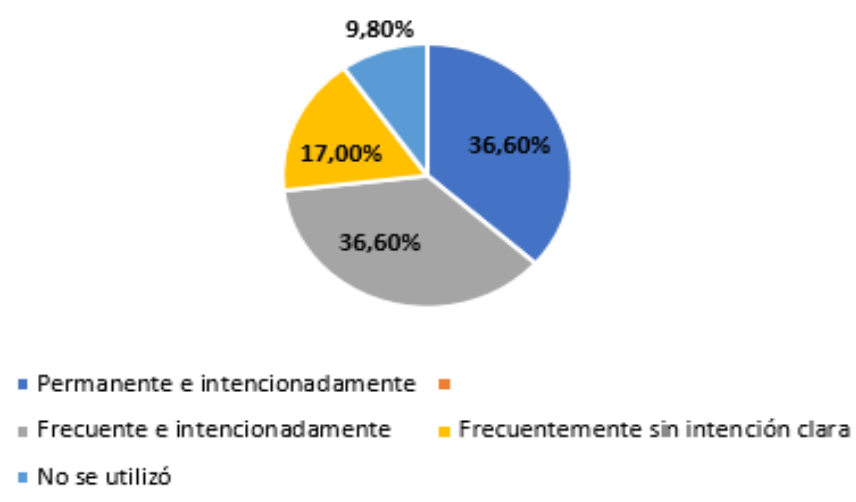
Como podemos apreciar el 36.6\% de los informantes reporta que si se utilizan los resultados de los procesos evaluativos para modificar las prácticas docentes de manera permanente e intencionada. Igual porcentaje informa hacerlo frecuente e intencionadamente. Un 17\% de los profesores de la muestra reconoce usar la información de forma frecuente, pero sin una intención tan definida. El 9,8\% restante no utiliza los resultados de los procesos evaluativos para modificar su práctica pedagógica.

\subsection{ANÁLISIS CUALITATIVO DE LA INFORMACIÓN}

En esta sección presentaremos y analizaremos la información obtenida mediante las preguntas abiertas de la encuesta aplicada en línea.

\subsection{EJE TEMÁTICO: CAMBIOS EN LA EVALUACIÓN POR LA EDUCACIÓN EN LÍNEA}

Uno de los elementos al que hacen referencia los informantes son los cambios que debieron implementar tanto en las metodologías de enseñanza como en los procedimientos evaluativos. "El cambio fue radical en cuanto a exigencia, pertinencia y contextualización. Progresivamente, el proceso de enseñanza-aprendizaje fue decantándose en exigencias mayores, desafiantes y, en algunos casos, estresantes. Pasamos desde la desconfianza y el manejo básico de programas, aplicaciones y estrategias para enseñar que eran arcaicas a unas más dinámicas, atingentes al nuevo siglo, aunque con la incertidumbre del grado o nivel de aprendizaje efectivo de nuestros estudiantes". (Informante 19)

Del mismo modo, los informantes declaran haber tenido más problemas en recoger las evidencias de aprendizaje al realizarlo de forma remota, más que cuando el proceso de enseñanza - aprendizaje se realiza de forma presencial. Estas dificultades no solo remiten al uso de nuevas plataformas educativas, o adaptación de los instrumentos de evaluación, sino que, a la veracidad de los resultados recogidos, así lo menciona el Informante 24 "Las evaluaciones pasaron a ser en plataformas online, o enviadas a la casa, sabiendo que la información recibida no era 100\% fidedigna a lo que el/la estudiante sabía."

Otro aspecto que los docentes debieron adaptar fue el uso de plataformas digitales dentro del proceso de enseñanza como de los procedimientos e instrumentos de evaluación "Cambió el formato de evaluación. Debimos aprender sobre diferentes plataformas para evaluar los aprendizajes online." (Informante 18)

Varios de los informantes señalan que en algunas áreas se pudo innovar en el uso de instrumentos de evaluación "Se hicieron podcast con previa explicación de rúbrica" (Informante 13) mientras que en otras áreas se dificultaron los procedimientos debido a los niveles de escolarización de los estudiantes, como la falta de herramientas digitales, tanto de los profesores como de los educandos y se debió incorporar a otros agentes educativos, la familia, para recoger evidencias del aprendizaje de los alumnos y sus intereses. Así lo señala el informante 21 "Evaluábamos a los niños de jardín infantil a través de 
registros cualitativos. Y mantuvimos comunicación semanal con los apoderados. Se consideraban las necesidades e intereses que arrojaban los registros a la hora de planificar".

A la luz de lo analizado, se puede concluir que en mayor o menor medida todos los informantes han debido modificar su práctica evaluativa, ya sea usando diferentes instrumentos que antes no utilizaban, así como modificando el nivel de participación del alumnado.

\subsection{EJE TEMÁTICO: FORMAS DE RETROALIMENTACIÓN}

En lo que a formas de retroalimentar se refiere, ocho informantes declaran no haber podido hacerla o en escasas ocasiones. "Muy difícil lograr una retroalimentación. No se hizo en mi caso." (Informante 32) Las formas de retroalimentación de aquellos profesores que sí lograron entregarla son muy variadas y diversas. Dentro de las respuestas de los participantes encontramos retroalimentación clase a clase, después de cada evaluación, de manera grupal, mediante informes escritos tanto a los padres como a los alumnos, a través de trabajo colaborativo, debates entre alumnos y comentarios breves del profesor a las respuestas erróneas de los alumnos en prueba

Quienes explicitan los procesos de retroalimentación asistidos por tecnología hacen referencia a video llamadas, el uso de dispositivos digitales, archivos subidos a drives en plataformas como Google o Seesaw, correos personalizados "Se enviaban retroalimentaciones individuales a través de un mail a cada alumno." (Informante 19), comentarios vía correo o WhatsApp y uso de secciones en plataforma creada específicamente para la entrega de retroalimentación: “Además, la plataforma con la que trabajábamos tenía una sección de retroalimentación en donde el profesor realizaba correcciones a cada estudiante, de forma individual, mediante la rúbrica correspondiente." (Informante 40)

En esta sección hemos podido apreciar que la retroalimentación ha sido un tema complejo, que un porcentaje de profesores no la logrado darla y que el resto ha recurrido, según sus posibilidades, a medios de comunicación social o a plataformas digitales más elaboradas para fines educativos.

\subsection{EJE TEMÁTICO: PARTICIPACIÓN DE LOS ESTUDIANTES EN EL PROCESO EVALUATIVO}

La rticipación de los estudiantes en los procesos evaluativos ha sido tradicionalmente escasa en la realidad chilena. Esta situación, a la luz de las respuestas de nuestros informantes, parece no haber cambiado en el contexto de la evaluación en línea. De hecho, 18 profesores señalan no haber incorporado a sus alumnos a los procesos de evaluación. Desde un directo "no" hasta respuestas más elaboradas como la del informante 15, dan cuenta de esta situación: "No, lamentablemente, el establecimiento es, además de academicista, vertical y con una implementación incipiente de prácticas pedagógicas donde se incorpore a las estudiantes en su proceso de evaluación." 
Las formas de participación descritas por los otros informantes son diversas y variadas. Ellas incluyen autoevaluación (la más frecuentemente mencionada), evaluación grupal, coevaluación "Se generaron instancias de coevaluación donde los pares podían comentar el trabajo y buscar puntos favorables y por mejorar que podían apreciar." (Informante 18), sugerencias para la evaluación, interacción con el docente y los pares, análisis de grado de avance, procesos de metacognición, ayudando a definir criterios de evaluación,

\subsection{EJE TEMÁTICO: INSTRUMENTOS DE EVALUACIÓN MÁS UTILIZADOS}

Consultados acerca de los instrumentos de evaluación más utilizados en el contexto ya descrito, los informantes entregaron las siguientes respuestas. Un grupo de nueve profesores informa haber realizado sus evaluaciones a través de medios tecnológicos como Google Forms, Classroom y Nearpod. La forma que toman estas evaluaciones son rúbricas, registros fotográficos, pautas de corrección, "Módulos de trabajo, guías de trabajo autónomo, pruebas tipo examen a través de aplicaciones" (Informante 3), guías, tareas, listas de cotejo, evaluación de informes, registros interactivos, exposiciones cortas, vídeos y evaluación escrita online.

Otros profesores informan haber usado portafolio, exposiciones, proyectos de integración, listas de cotejo, escalas de apreciación, rúbricas, interrogaciones orales, trabajos de investigación, “rúbricas para videos y presentaciones orales, pruebas de selección múltiple, pruebas de respuestas abiertas" (Informante 14), corrección de guías, podcast, ensayos, afiches, pruebas de alternativas, proyectos en equipo, creación de mapas conceptuales, rúbricas de metacognición, registros cualitativos, coevaluación, registros de observación en clases, fotografías y videos, producción de textos, exposición oral, disertaciones, corrección de tareas, pruebas de desarrollo, mapas conceptuales y organizadores gráficos.

Si bien los informantes no explicitaron que estos instrumentos fueron utilizados como medios de evaluación de los aprendizajes en líneas, por la situación sanitaria del país al momento de la evaluación, podemos inferir que sí fueron utilizados en esa modalidad. Si bien nos es parte de los objetivos de nuestra investigación indagar acerca de la precisión conceptual de términos asociados a evaluación de los aprendizajes, podemos sugerir que no existen límites nítidos entre tipo, forma, técnica, medio e instrumento de evaluación.

\subsection{EJE TEMÁTICO: INSTRUMENTOS DE EVALUACIÓN MÁS EFICIENTES}

Muchos de los instrumentos informados como utilizados se repiten en el listado de los más eficientes. Este hecho conlleva cierta lógica, ya que no tendría sentido usar instrumentos ineficientes; no obstante, la Informante 1 considera que "ni uno fue efectivo." De todas maneras, este fue un caso aislado. 
El resto de los profesores encuestados señala entre los instrumentos eficientes a las rúbricas de evaluación, los registros fotográficos, las guías de trabajo autónomo, las exposiciones orales, los formularios Google, las escalas de apreciación, las exposiciones, los podcasts, las evaluaciones parciales, las evaluaciones sumativas, las interrogaciones orales, los trabajos de producto, las pruebas escritos de desarrollo, documentaciones, registros, coevaluación, guías, test de pregunta abierta, trabajos de investigación y su presentación, rúbricas de autoevaluación, “la producción de textos guiada” (Informante 25), listas de cotejo, ensayos, fotografías de trabajos, pruebas de alternativa, portafolio, organizadores gráficos, evaluación de proceso, evaluación sumativa y evaluación acumulativa. Volvemos a identificar esta falta de precisión conceptual de términos asociados a la evaluación educacional.

Hacemos mención aparte al Informante 31 quien fue el único que otorgó a los estudiantes un rol en la eficiencia del instrumento a sus alumnos: "Todas aquellas que el propio estudiante adapta según sus intereses."

\subsection{EJE TEMÁTICO: RAZONES QUE DAN CUENTA DE SU EFICIENCIA}

Si bien todos los informantes identificaron instrumentos de evaluación considerados eficientes por ellos, no todos entregaron las razones para sustentar dicha valoración. En esta sección presentaremos dichas razones asociadas a su respectivo instrumento.

Al igual que en el eje temático anterior, la Informante 1 considera que no hubo instrumento eficiente alguno. Una vez más fue el único caso con un planteamiento así.

A juicio de los informantes, los registros fotográficos resultan eficientes porque los alumnos los disfrutan y pueden compartir sus trabajos con el curso. Además, permiten evidenciar de manera empírica los resultados de los estudiantes.

Las guías de trabajo autónomo también son consideradas como eficientes por los profesores dada la facilidad de acceso y su universalidad. Adicionalmente permitirían "una retroalimentación inmediata a la cual se suman las observaciones del docente de asignatura, por ende, los estudiantes tienen claro los elementos que deben mejorar para la siguiente instancia evaluativa." (Informante 3) La posibilidad de visualizar el estado de escritura de letras y números es otra razón recogida para la eficiencia de las guías.

El uso de videos y Podcast también resultó eficiente para varios encuestados. Dentro de las razones que aportan encontramos comprometer al alumno con su aprendizaje, la posibilidad de utilizar rúbricas y de acompañar durante el proceso de generación del video y la evidencia que entregan.

Respecto a las exposiciones y presentaciones orales, los informantes valoran la rápida entrega de resultados y la motivación y concentración que generan. Los formularios Google utilizados para fines evaluativos son considerados eficientes por la rapidez de evaluación, "la corrección semiautomática y personalización a cada estudiante” (Informante 9) y el hecho de que lleguen a todos los estudiantes. 
La rapidez y facilidad de evaluación y la consiguiente optimización del uso del tiempo, es la razón que justifica la eficiencia de las escalas de apreciación. Además, permitirían sistematizar las mejores prácticas con los distintos niveles.

Las rúbricas, por su parte, también son mencionadas con cierta frecuencia como eficientes por los encuestados. Las razones para aquello son: "exponen claramente los criterios a evaluar, de dominio público, foco puesto en el aprendizaje” (Informante 10), permiten monitorear y retroalimentar con rapidez, permiten guiar a los estudiantes y la claridad que cada alumno tiene acerca de lo que se evalúa.

Los trabajos grupales de investigación también son informados como eficientes, principalmente porque resultan entretenidos para los alumnos, posibilitan demostrar el conocimiento adquirido, permiten evaluar varios indicadores a la vez, facilitan una buena actitud de los alumnos, quienes, a su vez, "generan redes de apoyo entre ellos." (Informante 15) Otras razones esgrimidas por los encuestados son que los trabajos de investigación propician el conocimiento de la realidad de los integrantes del grupo, fomentan la creatividad, permiten que los estudiantes elijan un tema de su interés y "facilitan cotejar el desarrollo del proceso." (Informante 35)

La eficiencia de las pruebas de alternativa queda sustentada en su rapidez de corrección y en la familiarización que tienen los estudiantes con ellas. Respecto a las pruebas escritas o de desarrollo, quienes las consideran eficientes señalan que evidencian no solo el manejo del conocimiento sino también la capacidad de expresar por escrito conceptos e ideas con claridad. El Informante 38 parece concordar: "La producción escrita revela de forma más evidente que cualquiera el nivel alcanzado en las habilidades mentales puesto que su propia lógica implica ciertas formas de organización del pensamiento que son de índole superior, sea esto por lógica, retórica, análisis, reflexión, dialéctica, etc." Otra razón presentada es la posibilidad que las pruebas de desarrollo entregan para observar la coherencia de los textos y la comprensión profunda de los mismos.

Los encuestados consideran que las rúbricas de evaluación son eficientes debido a que permiten retroalimentar y señalan el grado de consecución del objetivo. Respecto a las listas de cotejo ellas son consideradas eficientes porque "responden de una u otra manera al trabajo realizado con los niños y niñas, pudiendo entregar un panorama general de sus aprendizajes y también permitiendo una visión específica en caso de necesitarlo." (Informante 27) Su facilidad de aplicación sería otra razón de su eficiencia.

Las tareas como instrumento de evaluación permiten, a juicio de los informantes, valorar los procesos de desarrollo y las etapas que los estudiantes van cruzando. Respecto al portafolio, el Informante 35 considera que es eficiente "porque una mayor participación de los estudiantes en cada una de sus etapas." 
La plataforma Nearpod es considerada eficiente por el Informante 29 debido a que ". fomenta la participación en clases virtuales y, simultáneamente, permite guardar registros de dichas participaciones en quizzes, preguntas abiertas, encuestas, pizarras colaborativas, etc." Todas estas actividades permitirían dar cuenta del proceso de aprendizaje de los estudiantes.

La autoevaluación si bien no es un instrumento de evaluación también es valorada por algunos informantes porque permite un momento personalizado con cada estudiante y porque permiten procesos reflexivos acerca del aprendizaje de cada estudiante. Algo similar ocurre con la coevaluación considerada eficiente por un informante debido a que llega a todos los alumnos.

\section{CONCLUSIONES Y PROPOSICIONES}

Los profesores han experimentado serias dificultades al tener que afrontar los procesos pedagógicos y en especial los evaluativos de los aprendizajes en línea. Al trabajar desde sus casas, el diseño de instrumentos de evaluación se volvió aún más personal y, por lo tanto, menos colaborativo. Lo mismo ocurre con la escasa participación de los alumnos en dichos procesos. En búsqueda de soluciones, los encuestados privilegiaron instrumentos de fácil aplicación a distancia, sencillos de corregir y que tuvieran algún impacto en el grado de motivación de sus estudiantes.

Dentro de los principales cambios que los profesores debieron realizar figuran cambios en las prioridades donde la flexibilidad, la participación activa de los estudiantes y la asistencia virtual adquirieron mayor relevancia que el trabajo con contenidos o la cobertura curricular. En este mismo ámbito, la evaluación formativa pareciera adquirir más importancia que la sumativa.

Otra línea de cambio producto de la enseñanza y evaluación virtual, dice relación con el soporte tecnológico. Surge la necesidad de modificar metodologías, de apoyarse en plataformas y de un mejor entrenamiento en el uso pedagógico de las mismas. Los procedimientos evaluativos también sufrieron variaciones, al incorporar los profesores distintos instrumentos en formato digital. La posibilidad de evaluar y retroalimentar adecuadamente también genera problemas con los cuales los docentes han tenido que lidiar, generando sensaciones de agobio y sobre demanda.

De lo expuesto anteriormente, surgen distintos desafíos de los que los establecimientos educacionales, los profesores y las autoridades respectivas deberán hacerse cargo. Primeramente, la insuficiente preparación de los profesores para enseñar y evaluar en entornos virtuales, crea la necesidad de implementar sistemas de capacitación para fortalecer su manejo tecnológico para fines educativos.

Las reducidas instancias de trabajo colaborativo entre colegas, convoca a una búsqueda urgente de espacios de reflexión y colaboración en línea que permitan, a la luz de los resultados de los estudiantes, mejorar las prácticas pedagógicas de los profesores y los procedimientos de necesaria retroalimentación a los niños y jóvenes a su cargo. 
La baja participación de los estudiantes en los procesos evaluativos es otro desafío pendiente. En la búsqueda de una evaluación más auténtica de los aprendizajes que entregue una visión más holística e integradora de los resultados, es necesario entregarles a los alumnos un rol, junto a un espacio de colaboración. De esa forma, se sentirán más motivados a hacerse responsables de su proceso de enseñanza aprendizaje y los profesores lograrán resultados más confiables y significativos para poder evaluar su propia práctica a partir de los resultados de sus estudiantes. 


\section{BIBLIOGRAFÍA}

Ambrose, S., Bridges, M., Di Pietro M., Lovett, M. y Norman, M. (2010). How Learning Works: Seven Research-Based Principles for Smart Teaching, USA: Jossey-Bass

Amua-Sekyi, E. (2016). Assessment, Student Learning and Classroom Practice: A Review, Journal of Education and Practice 7 (21): 1-6

Bingham, T. y Conner, M. (2010). The New Social Learning, New York: Penguin Random House

Brown, P., Roediger, H. y McDaniel, M. (2014). Make It Stick: The Science of Successful Learning, USA: Library of the Congress

CEPAL (2020). América Latina y el Caribe ante la pandemia del COVID-19: efectos económicos y sociales. https://www.cepal.org/es/publicaciones/45337-america-latina-caribe-la-pandemia-covid-19efectos-economicos-sociales

Dixson, D. y Worrell, F. (2016). Formative and Summative Assessment in the Classroom, Theory into Practice 55 (2): 153-159

Driscoll, M. (2005). Psychology of Learning for Instruction, USA: Pearson

Engzella, P, Freya, A. y Verhagena M. (2021). Learning loss due to school closures during the COVID19 pandemic, PNAS 18 (17): 1-7

Gagne, R. (1965). The conditions of learning, New York: Holt, Rinehart and Winston

Gore, J., Fray, L. Miller, A., Harris, J. y Taggart, W. (2021). The impact of COVID-19 on student learning in New South Wales primary schools: an empirical study, The Australian Educational Researcher, March Hernández, C., Pérez, B. y Quiroz, C. (2021). Evaluación del Método de Aprendizaje Basado en la Investigación (MABI) en el CICS UMA-IPN, South Florida Journal of Development, Miami 2, (5):70857092

Lecrecq y Cabrera (2014). Ideas e innovaciones. Dispositivos de evaluación de los aprendizajes en la educación, Santiago de Chile: Editorial Universitaria

Mateo, J. y Martínez, F. (2008). Medición y evaluación educativa, Madrid: Editorial la Muralla

Mayer, R. (2003). Learning and Instruction, New York: Pearson

Murillo, J. y Duk, C. (2020). El Covid-19 y las brechas educativas, Revista Latinoamericana de Educación Inclusiva 14(1): 11-13

Pimienta, J. (2008). Evaluación de los aprendizajes. Un enfoque basado en competencias, México: Pearson

Sardareh, S. y Mohd Saad, M.R. (2013). Defining Assessment for Learning: A proposed definition from a sociocultural perspective, Life Science Journal 10 (2): 2493- 2497 
Soland J., Kuhfeld, M., Tarasawa, B., Johnson, A., Ruzek, E. \& Liu, J. (2020). The impact of COVID-19 on student achievement and what it may mean for educators, https://www.brookings.edu/blog/browncenter-chalkboard/2020/05/27/the-impact-of-covid-19-onstudent-achievement-and-what-it-may-meanfor-educators/

UNESCO (2020). Impacto de Covid-19 en la Educación - Datos. Paris, Francia: UNESCO. https://es.unesco.org/covid19/educationresponse

Western and Northern Canadian Protocol for Collaboration in Education (2006). Rethinking Classroom Assessment with Purpose in Mind, Canada: Manitoba Education, Citizenship and Youth Cataloguing in Publication Data 